\title{
Simulation of Energy Selective Signal Amplification in Gas Environment of Variable Pressure SEM
}

V. Neděla, ${ }^{*}$ I. Konvalina, ${ }^{*}$ B. Lencová, $* * *$ and J. Zlámal**

* Institute of Scientific Instruments ASCR, v.v.i, Královopolská 147, 61264 Brno, Czech Republic

** Institute of Physical Engineering, Faculty of Mechanical Engineering, Brno University of Technology, Technická 2, 61696 Brno, Czech Republic

High pressure of gas mostly water vapour, with pressure range from $0.1 \mathrm{~Pa}$ to $80 \mathrm{~Pa}$ in the first differentially pumped chamber and $10 \mathrm{~Pa}$ to over $2000 \mathrm{~Pa}$ in the specimen chamber is used in variable pressure scanning electron microscopes (VP-SEM) such as our experimental AQUASEM II equipped with a combined YAG-BSE and ionization detector of SE, see Figure 1. The gas environment causes unwanted primary electron beam diffusion, but it also supports gas ionization cascade amplification of the signal of secondary electrons (SE), accelerated by the applied field of detection electrode of the ionisation detector [1].

Investigation of new detection principles as well as development of existing detection systems for high pressure conditions of VP-SEM require the support of suitable calculation software including gas ionisation effect. In order to understand the signal generation in the gas environment, the program EOD [2], used mainly for field computation and ray tracing, has been extended with a plugin module allowing to include collision phenomena (electron gas interactions). The time-consuming Monte-Carlo computations of secondary, environmental and backscattered electrons with small time step use ionisation cross-sections from NIST database. Dissociation, vibration and momentumtransfer cross sections of $\mathrm{H}_{2} \mathrm{O}$ molecules are also included in the calculation algorithm. The secondary (SE) and backscattered (BSE) electrons emitted from the specimen and detected by the ionization detector were calculated for $\mathrm{SE}$ energies $\mathrm{E}_{\mathrm{SE}}=1,3,5,10$ and $20 \mathrm{eV}$ and BSE energies $\mathrm{E}_{\mathrm{BSE}}=3,5,10,15$ and $18 \mathrm{keV}$ and for polar angle from $\Theta=0^{\circ}$ to $90^{\circ}$ with a step $1^{\circ}$. Totally 2000 trajectories were simulated for each energy of emitted electrons.

Dependencies of signal amplification in gas environment on the water vapor pressure and on the energy of electrons going through the gas, shown in figure 2, were calculated using the EOD software equipped with a Monte Carlo plug-in. It is seen that the highest amplification of the signal is for electrons with energy of $250 \mathrm{eV}$. The lowest amplification was obtained for signal with the highest energy of $20 \mathrm{keV}$. The results in Fig 2 describe electron-gas interactions, without the influence of real energy distribution of the signal electrons emitted from the sample. Computed results considerably depend on the geometry and parameters of the detection system as well as on the geometry of the specimen chamber of the microscope. Figure 2 also shows that the maxima of amplified signal are shifted to a higher pressure with the increase of electron energy.

References

[1] G.D. Danilatos, Foundations of Environmental Scanning Electron Microscopy, Academic Press, Sydney, 1988.

[2] B. Lencová, J. Zlámal, A new program for the design of electron microscopes. Physics Procedia 1 (2008) 315-324.

[3] This work was supported by Grant Agency of the Czech Republic, grant No. GAP102/10/1410 


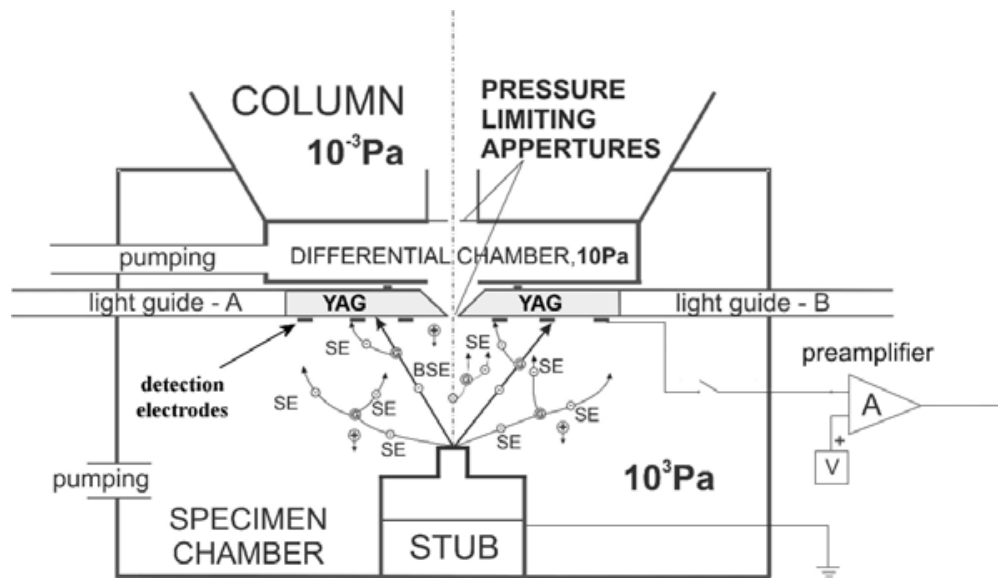

FIG. 1. Configuration of the combined YAG-BSE detector and ionisation detector of SE in VPSEM AQUASEM II.

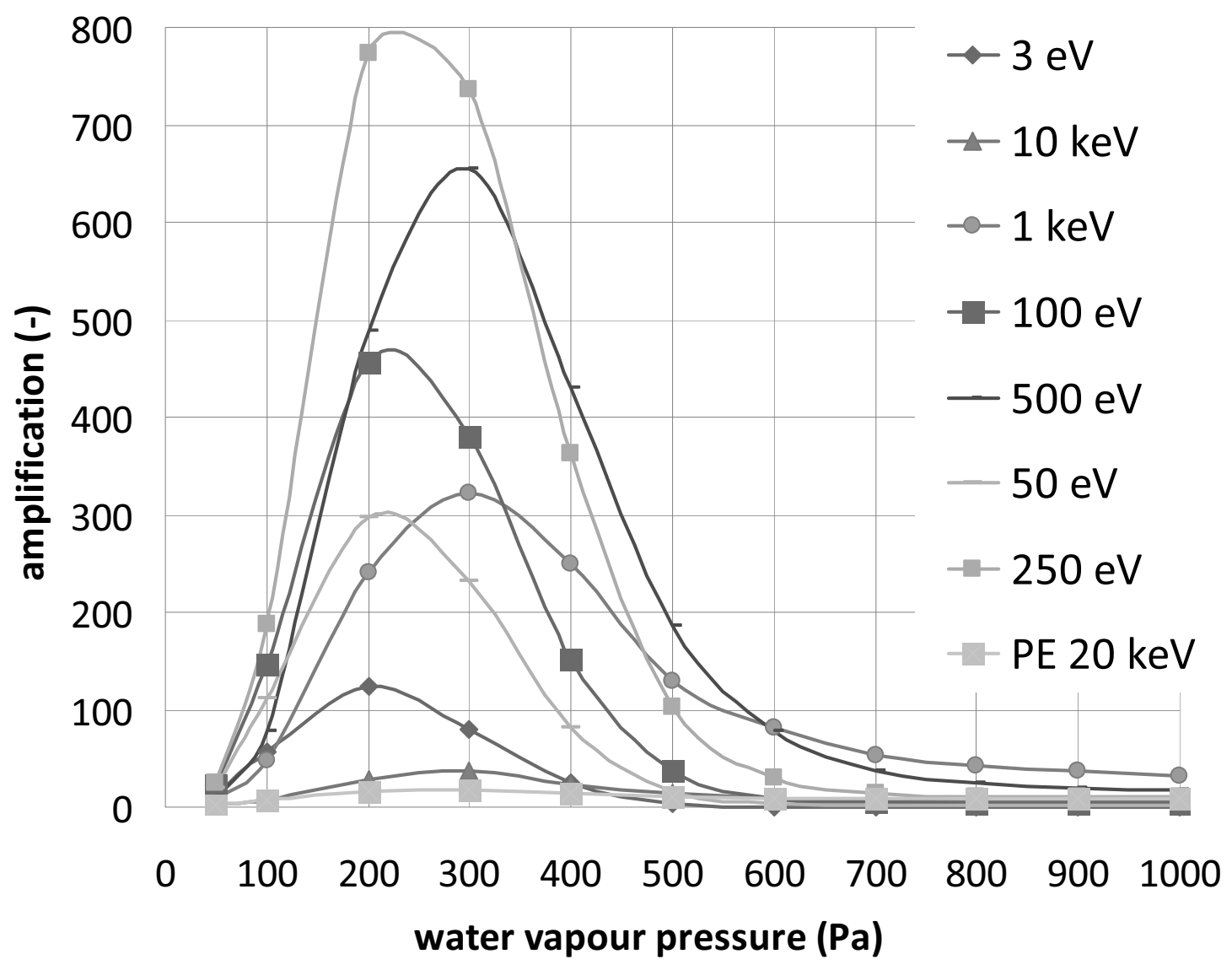

FIG. 2. Dependencies of signal amplification in gaseous environment on the water vapor pressure and on the energy of electrons. 2000 electrons were simulated for each value of pressure. Sample to detection electrode distance $6 \mathrm{~mm}$, potential on the detection electrode of ionisation detector $300 \mathrm{~V}$. 